Bull. Mater. Sci., Vol. 4, No. 3, May 1982, pp. 347-356. (C) Printed in India.

\title{
Current materials sciences in space activities in Grenoble
}

\author{
YVES MALMEJAC \\ Commissariat A L'Energie Atomique, DMG/LES, Grenoble, France
}

MS received 23 January 1980

\begin{abstract}
Abstrabt. The "Laboratoire d'Etude de la solidification" (L.E.S.) in Grenoble is currently in charge of a significant number of various space experiments. They are 13 for the time being belonging to 7 programmes in cooperation with 5 international or national space agencies. It stands to reason that these 13 projects are not concerned with 13 entirely different problems. The six scientific problems that L.E.S. is trying to solve by operating in space conditions were not intraduced as new activities, but rather as the best solution to solve many problems that were encountered in the frame of the classical ground-based research activity of this Laboratory. They are: (i) Thermomigration studies in liquid alloys $(\mathrm{Sn}+\epsilon X)$, (ii) Nucleation of eutectics (Ag-Ge, Au-Si), (iii) Unidirectional solidification of eutectics ( $\mathrm{Al}-\mathrm{AlCu}, \mathrm{Al}-\mathrm{Al} \mathrm{Ni}, \mathrm{Ag}-\mathrm{Ge}$ ). (iv) Unidirectional solidification of solid solutions (Ge-Si, $\mathrm{Al}-\cdot \mathrm{Cu}, \mathrm{Sn}-\cdot \mathrm{Pb}, \mathrm{Bi}-\cdot \mathrm{Sn}, \mathrm{Ge}+\epsilon \cdot \mathrm{Ga}$ ). (v) Unidirectional solidification of emulsions (Al-In). (vi) Unidirectional solidification of foams (Al-Zn).

Amongst these thirteen projects, three will use or have used rackets, three have used the Salyut-6 equipments, one will be operated as an automatic experiment aboard the Space Transportation System (the Space Shuttle), and the six remaining ones will be realized aboard the European Spacelab. Eleven experiments will use common facilities, one of those the low gradient heating facility (LGHF), being built in Grenoble: this equipment will be used by us in three experiments of the F.S.L.P. (first spacelab payload) and by two other European investigators.
\end{abstract}

Keywords. Thormomigration studies; nucleation of eutectics; solidification of eutectics; solidification of emulsions.

\section{Thermomigration stadies}

Two projects a re currently developed on these topics, one in the frame of the European Space Agency Pragramme and the ather one in cooperation with the Swedish Space Corporation Rocket programme.

Many experiments that were previously performed in Grenoble had demanstrated the importance of thermomigration mechanisms with regards to crystal growth. Although it is a second order effect, and is thus more sensitive than chemical diffusion to convection or segregation, it affects the stabilizing or destabilizing mechanisms of the interface as well as the distribution of the components in both liquid and solid phases. Its importance could be invaked to explain these features in processes such as Bridgman solution, or cutectic growth. 
This phenomenon cansists of the segregation of the components of an alloy, or of the isotopes of a pure element, under a given temperature gradient. It was first studied and vesd in gases, but experimental difficulties invalved in the use of liquid metals and the rather low amplitude of concentration variations usually observed, have limited investigations in this field. Effectively, these concentration variations do not usually exceed some thousand $\mathrm{ppm}$ which is difficult to detect, but may be of the greatest importance in deciding some critical behaviours (constitutional supercooling for instance).

Usually, one can abserve that when an alloy of initially hamogeneous composition is held for long enough in a temperature field, the system will advance to a steady state of minimum entropy production, for which transpart fluxes are zero. Once the steady state has been achieved, concentration variations provide the heat of transport as a function of the temperature gradient.

The development of solidification studies has caused an increased intercst in this phenomenon, because nearly all solidification processcs need such a temperature gradient. Thus we must observe, in the liquid region, opposed composition changes in both the coldest and the hottest regions. In the neighbourhood of the interface there will be a relative modification of the stable growth condtions, because these new fluxes will have to be algrebraically added to the classical solute-rejection fluxes created by the moving interface.

As often as nat, such changes are undesirable ; even under normal conditions, they are drastically opposed by gravity-induced convection or segregation. The effects will be increased in low-gravity environment in space. There is also a decrease in convective heat exchange ; thus increasing the value of the driving force i.e., the temperature gradient. Consequently we can assume that the space environment would increase the influence of thermadiffusion in every solidification pracess.

As already indicated, some previous investigations have demonstrated its importance, but they have also shown the lack of experimental data to use in calculations. For this reason, we began 8 years ago to measure these second-order effects in various metallic liquid systems, and we were able to observe the diffculty of performing accurate measurements : when operating in a vertical position to eliminate thermal convection, a gravity-induced segregation is created and vice versa. We therefare cansidered it valuable to carry out a precise measurement of some heats of transport in ideal $0-\mathrm{g}$ conditions.

The interest will be two-fold : In the first place it will be possible to determine their exact influence in every crystallization process under $1-\mathrm{g}$ or $0-\mathrm{g}$ canditions ; secondly we shall gain a more accurate determination of the Sorct parameters allowing us to analyse their evolution as a function of both temperature and concentration. This will result in a better understanding of general thermodiffusion phenomena in metallic liquid melts. In particular, we hope to refine the demonstration we published recently concerning the existence of two contributions to the glabal heat of transpart : The first (électronic) contribution is the result of the interactions between the conduction electrons of the elements and it varies as the square of the absolute temperature. The second (intrinsic) contribution is the result of the collisions between the ions and it varies as the absolute temperature. 
In another respect, we believe that an accurate enough theory of this phenomenon for the ideal $0-\mathrm{g}$ experiments will later allow the calculation of the migration direction and of the order of magnitude of the transfer heat of any solute in any solvent, for any temperature, and thus provide a foreknowledge of any experimental crystal growth modifications, either in $0-$ gor $1-\mathrm{g}$ conditions. The improved theory so obtained will allow also an improvement in the experimental conditions of some particular crystal growth mechanisms.

For instance, in the classical situation of the liquid epitaxial solution growth of some semiconducting compounds, an accelerated supply to the crystal by those elements which are the least abundant will be achieved through thermadiffusion fluxes. This will be achieved by choosing the initial composition on the right side of the compound, and by defining a level of temperature such that the thermodiffusion filuxes which are directed towards the coldest regions, and thus towards the interface, are in the feeding direction fallowed by the least abundant component. Such experimental conditions will be more efficient in space than on the ground, and will allow the crystal grawth rate to be increased while opposing the constitutional supercoaling destabilizing mechanism.

To achieve these results, the experimental device we developed will be able to suppress any source of residual convection (especially Marangoni flow), agitation, or segretation. On the other hand, we have defined some liquid systems, taking into cansideration the possibility of separating mass effects, from purely electronic effects, which implies knowledge of critical physical properties (structure factor, electrical resistivity, thermoelectric power, thermodynamical factor, and the possibility of using low-activity radioisotopes).

\section{Nucleation of eutectics}

Eutcetics must be considered as the standard example for the theary of solidifisation. Two systems are studied, the behaviour of which cauld be explained by the existence of clusters inside the liquid phase in the vicinity of the eutectic point, as demonstrated by an extensive study of the $\mathrm{Ag}-\mathrm{Ge}$ eutectic liquid alloy in Grenoble. The lifetime of such clusters in a terrestrial environment must be shorter due to convection currents and to gravity segregation effects. Thus a low gravity environment should lead to a higher tendency of these clusters to behave as nuclei.

Furthermore, the comparison of maximum undercoolings obtaincd in crucibles on the ground and in space, and in space with and without crucibles, correlated with same abserved discrepancies in the wetting properties that were already abserved when operating in space, ought to help in the analysis of heterogeneou nucleation : The main disadvantage during experiments on earth resides in the risk of such substrate effects essentially at the level of the crucible containing the alloy and in the vicinity of su bmicroscopic impurities thet will be more efficient when convection is present. Another ground disadvantage is linked to heterogencities in composition that are due to the sedimentation phenomena: They are a.pt to occur within each single phase having a polycomposition and they may induce parasitic precipitation phenomena. 
Thus three experimental runs will be necessary for each composition (eutectic, hypo- and hypereutectic) and any modifications to the morphology and the dimensions of the phases present in the resulting solids will also have to be compared.

We endeavoured to summarize the possible influence of zero-gravity on factors affecting nucleation, from the classical theories :

(i) no zero-gravity influence on thermodynamic driving forces (embryo size and shape, bulks and surface free energy, valume of material),

(ii) high zero-gravity influence on wetting of substrates and foreign particles by eutectic liquids,

(iii) high zero-gravity influence on liquid motion,

(iv) high zero-gravity influence on local supersaturation, gas content, soluble impurities.

This enumeration summarizes the reasons we had to study eutectic nucleation in $0 \rightarrow \mathrm{g}$ conditions. It is obvious that they are only the results of the next $0 \rightarrow \mathrm{g}$ experiments that will allow to verify those purely speculative ideas.

\section{Unidirectional solidification of eutectics}

Unidirectional solidification of an eutectic metallic system leads to a two phase structure, either lamellar, or fibre-like. The microstructure is anisotropic and oriented in the solidification direction. Those eutectic structures are unusual because, when the two phases have judiciously ohosen 1 hysical or chemical properties the material can enjoy very special properties. Unfortunately, the result af an eutectic solidification is not usually a thearetical periodic network with an infinite size ; the actual structures entail some defects which are the result of instabilities of bath the extrinsic and intrinsic mechanisms affecting the growth.

Thus, during the growth, several mechanisms are involved :

(i) heat transfer between liquid and solids,

(ii) mass transfer by diffusion and convection,

(iii) solid/solid interface formation,

(iv) kinetic behaviours of each phase.

In another light, the parameters governing the eutectic structure are both the intrinsic thermodynamic properties of the eutectic system ;

(i) volume ratio of the phases,

(ii) phase diagram behaviour,

(iii) growth anisotropies,

(iv) phase crystallography,

and the extrinsic experimental growth parameters :

(i) growth rate,

(ii) thermal gradient in the liquid,

(iii) alloy composition.

Our project is to consider how the spatial environment gives the possibility of better access to the different mechanisms involved during the global process.

In the first place are the mechanisms affecting the volume phases, the first one being the disappearance of the tendency to segregate in the case of large differences 
in the densities of the camponents that introduces a vertical gradient of concentration when operating in ground conditions. The second mechanism will be the disappearance of convection phenomena within the liquid resulting in a decrease in the driving forces for heat and mass transfers but also in a greater regularity of the fluxes. The process of chemical diffusion being affected by diflusion each time the diffusion boundary layer will be greater than the convection baundary layer, by operating in space without any convection, such a situation becomes impossible, and consequently, all the phenomena governed by diffusion (redistribution of the solutes, morpholagical stability, size of the structures) will be unaffected.

The first advantage will be reinforced by an other one since in parallel the length of the chemical initial transient stage in a solidification experiment will be shorter at $0-\mathrm{g}$ than at $1-\mathrm{g}$.

Furthermore, by eliminating thermoconvection, disturbances of the heat and mass fluxes that are generally difficult to control are also suppressed. And it is known that losal thermal fluctuations at the salidification interface are responsible for punctual creation of structural defects in the salid being formed (lamellar faults or branching of eutectic structures). The absence of fluctuations at the interface would therefore be favourable for more regular growth and an improvement in the structural perfection of the solid may be expected in space if any other perturbation of the growth rate does not occur.

Interfacial mechanisms are the second kind of mechanisms involved during growth. In fact, depending on the atomic structure of the solid-liquid interface (rough or facetted), we can expect that growth will be mare or less facilitated. In the facetted case crystallization of the alloy will not occur at thermodynamic equilibrium, as most theories assume, but a quantity of solute, other than that anticipated at equilibrium will be rejected ahead of the interface giving rise to a flux of kinetic origin. Improved control over the mass fluxes should make it possible to apprehend the kinetic eftects of attachment of the atoms at the liquidsolid interface which are generally masked or disturbed by the convection in the liquid. The relatively weak kinetic flux can only be detected and measured if all the other fluxes are well controlled, which will be easily achieved in space.

However experiments performed in a space environment will have to take into account new or non attenuated phenomena that are likely to appear. The effect of such phenomena could result in a modification of the experimental parameters:

(i) modifications of thermal gradients in the liquid at the interface,

(ii) modifications in the local compasition of the liquid,

(iii) modifications in the interface displacement velocity.

In the light of this preliminary analysis, we could define the type of experiments that should be attempted under microgravity conditions in order to identify the role of each of these mechanisms and in order to check the exact influence of each of the parameters.

We do not expect immediately any spectacular result such as the achievement of a perfect eutectic structure, the achievement of which will necessitate at first the imf rovement of the control of the three parameters $G_{L}, G_{S}$ and $R$ that are linked inside the general heat flux equilibrium equation. Microgravity experiments were rather considered as a powerful means for apprehending the various 
mechanisms intervening in the solidification of eutectic alloys. If such experiments have been sufficiently well-defined (both chemical and thermal parameters of academic eutectic systems being accurately determined by supplement experiments), they will provide some answers in reply to the theoretical questions that are still outstanding.

\section{Unidiroctional solidification of salid solutions}

The interest of microgravity experiments to study the essential problem of the transition between stable and unstable growth interfaces was the main reason of this activity.

There are todate two kinds of theoretical approaches to this problem :

(i) The most simple theory (Chalmers and Tiller) only considers the destabilization mechanisms of the liquid phase in the vicinity of the growth interface.

(ii) The more elaborate theories (Mullins-Sekerka, Länger) consider the destabilization of the real interface.

Many ground experiments were performed with the aim of determining the limit of stability of planar interfaces and also to demonstrate the type of steady state structure resulting from destabilization but they did not r rovide sufficiently detailed information on conditions prevailing on the system during destabilization mainly in what concerns the nature (diffusion or convection) of transport phenomena within the liquid phase. Thus it appears diffcult to rely entirely on the quantitative analysis of such experiments that were not able to separate the influences of these two theories. The reasons for such a failure are as follows :

(i) To successfully study the liquid instability, we need to know with a high level of accuracy the values of the solutal transport fluxes in front of the solid interface. Whatever the care taken over current ground conditions (stabilizing thermal gracients, use of magnetic fields) the fluxes of chemical diffusion will remain modified by an unknown amount due to the effect of convection.

(ii) The random residual chænges due to these convective fluxes hide all the second order phenomena that are of primary importance to the study.

(iii) By definition, destabilization occurs in a transient made since it consists in the transition from one particular state to another particuls.r state. Because all the experimental studies have only taken inta account the presumed steady state which follows destabilization, they all obtained excess values for stability criteria.

With such difficulties, it is normal to expect disagreement between results abtained on earth and the theoretical models. Thus the values of the wavelengths of the stable fluctuations previewed by theary were never verified. It is the presence of uncontrolled fluctuations inside the liquid phase that perturbs the solute redistribution in the vicinity of the interface and that risks masking phenomena less efficient than bulk diffusion, such as those dependent on the interface propertics (capillary and kinetics). They are precisely these less efficient phenomena which we intend to study by ensuring the three following ideal conditions,

(i) Concerning bulk behaviour : good cantrol of the solute and heat flows inside the liquid phase. Such a control supposes the existence of purely diffusional flows, without the presence of any convective flow. 
(ii) Also concerning bulk behaviour : there must be no hydradynamic instability of the liquid phase that would risk initiating, or even imposing any particular morphology on the destabilized interface.

(iii) Concerning interfacial behaviour : absence of any thermal disturbances that would entail a modification of the interfacial properties or masking of related phenomena.

The resulting ideal conditions are available only by using zero gravity experiments. We decided to select academic metallic systems allowing us to suppress any major uncertainty in the determination of the necessary physico-chemical parameters. Two of our four experiments were flawn aboard SALYUT VI and the past-flight analysis work began a few weeks ago in Grenoble. We are also planning to use such experiments to study the problems of striations, our aim being to demonstrate the existence of intrinsic striations that would be independent of any convection current.

The most particular of these investigations concerns the Ge-Si system which is one of the few systems showing complete solid and liquid miscibilities. This system was chosen for theoretical reasons :

(i) The marked tendeney of the liquid to segregate under gravity, which leads to an inhamogeneous liquid phase, and consequently, to an inhomogeneous solid phase.

(ii) The width of the twa-phase region which is the largest one in phase diagrams: it increases the distribution effects, and delays the onset of a growth steady mode.

(iii) Because of hydrodynamic fluctuations in $1-\mathrm{g}$ conditions, one may speculate that grains are generated by the resulting compositional changes.

The connection of these thrce reasons makes this material difficult to obtain on earth and thus there was a further type of interest to study its growth behaviour in space conditions.

\section{Unidirectional solidification of omulsions}

Due to the absence of buoyancy, the absence of gravity was a prerequisite in order to praduce regular compasite structures by solidifying metallic dispersions such as the ones thit result from metallic systems with their phase diagrams exhibiting miscibility gaps.

The simplest way to obtain the required microstructure is to achieve both the decomposition of the hamogeneous liquid and the solidification of the resulting emulsion in the course of the same controlled cooling of the sample under zerogravity. This procedure will be applied to the Al-In alloy in the frame of two space programmes NASA/SPAR and ESA/Spacelab.

Whatever the simplicity of the initial idea the method sets four fundamental problems :

(i) liquid decomposition of the initially homogeneous alloy,

(ii) stabilization of the resulting metallic emulsion in zero-gravity,

(iii) monotectic solidification, and

(iv) final solidification of the emulsion in zero-gravity. 
The procedure that consists in controlling bath phase transformations, decomposition and solidification by means of a unidirectional thermal gradient will introduce various benefits :

- It will enable the steady state of phase transformation to be mare readily attained, and cansequently will provide the necessary condition for the formation of the desired regular structures.

- The stability of the metallic emulsion at $0-\mathrm{g}$ will be considerably diminished if the conditions of solidification are not controlled. In addition when a sufficiently high thermal gradient is imposed, the extension of the twophased zone is reduced and therefore the degree of coalescence of the emulsion may be reduced.

Whatever the former advantages many difficulties survive which have been listed :

- Physico-chemical interactions of the crucible material with the malten sample in its single and two-phases states?

- Required hamogenisation time of the initial single phase liquid?

- Nature of the nucleation mchanism of the new phase?

- Mechanisms governing the stability of the emulsion?

- Migration of the globules of second phase?

- Interaction of the globules with each other, with the interface in progress, and with the walls of the crucible?

- Efficiency of such collisions on coalescence?

This list of underlying problems illustrates the difficulties that have to be solved in order to achieve dispersoid salid allays from liquid emulsions obtained under microgravity conditions.

Previous experiments by other authors have demonstrated that the influence of gravity on buoyancy phenomena in the initial homogeneous liquid phase was not the only incriminating factor. A number of the pointed out problems will be more or less influenced when reducing the level of gravity and our proposed experiments attempt to separate the ways those various mechanisms do act either in one-gravity or in zero-gravity.

\section{Unidirectional solidification of foams}

Foaming is a typical liquid-state process consisting of the formation and dispersion of gas bubbles in or from a liquid continuum. To obtain a solid foam the bubbles and their distribution have to remain stable to the point of solidification. In an environment under gravity this requirement is only met by materials of high viscosity. The formation of solid foams from low-viscosity molten metal is not feasible unless very high solidification rates are used and these are inconsistent with the production of a homogeneous distribution of the gas bubbles. Under 0 -g conditions liquid foams will te more stable which will permit the growing solid phase to incorporate these bubbles. With respect to the foam maintenance it is necessary that the bubbles remain the right size in the right place, and that they do not coalesce before solidification. In a low-gravity environment, the situation will be much more favourable even thougb some geometris 
crowding will probably occur. The main problem is the formation of many small bubbles which, in contradistinction to the dispersion and the maintenance of the foam is not sensitive to gravitty. The two basic methods for the foaming of liquids are

(a) pressurization with extrinsically supplied gases, and

(b) intrinsic gas evolution from the liquid.

We have chosen a variation of this last method. A zine solid solution is partially remeited under low-gravity conditions in a sealed crucible. Well-defined thermodynamic states of the phases present will be reached after soaking in a fixed temperature field before the unidirectional solidification stage begins.

For the chosen growth conditions, a rapid increase of the zinc concentration in the liquid, ahead of the solid-liquid interface, will occur : the local thermodynamic equilibrium will breakdown and numerous bubbles of a zinc vapour pbase will appear locally by multiple nucleation and growth on the destabilizeddendritic interface. They will be incorporated in the growing solid as the release of their latent heat of evaporation decreases the local temperature of the melt, thus creating a relative displacement of the interface. We are at present studying the nucleating mechanisms, the evolution of the dimensions of these bubbles as a function of their wettability with the solid-liquid interface and of the geometry of the interface, and also the behaviour of such bubbles in the thermal gradient. We have already been able to verify the validity of the basic idea, and we believe that low-gravity conditions will prevent the tendencies to coalesce or to escape observed to date.

This experiment will be flown aboard the 1st Spacelab Mission.

\section{Conclusion}

Despite the numerous but now well-acknowledged faults of the initial materials processing activities, these preliminary experiments brought largely positive results, in particular because they helped us to understand better that the space environment was not only some kind of marvellous remedy for any ground-based problem. For us in Grenoble this space environment was considered from the beginning as a technical experimental facility at the service of research that is fundamental in nature and finality, in the same way as the centrifuges we are also using, and that gives us many good and complementary results.

Thus in this new perspective, and provided investigations are not restricted to isolated experiments, this extremely powerful means of investigation should procure precise answers, if the questions are sufficiently well-defined, to a number of fundamental problems that are still unresolved, concerning the true nature of certain mechanisms in the science of materials currently implemented on earth.

The most obvious merit of the experiments already accomplished in the field of metallurgy is no doubt the fact that they have drawn attention to a number of second-order phenomena, of which we were practically unaware until then, because they could not be quantitatively apprehended, in our earth environment. It is not unreasonable to jmagine that, due to the more ideal conditions prevailing 
in space, the fundamental metallurgical process consisting in the passage from a liquid to a solid stage, will at least be apprehended in its most general aspect of a non-equilibrium phenomenon, taking place in the non-steady mode. The much more precise understanding that will result from this analysis will then allow a much better control of the processing of solid materials, not only at microgravity, but also-and this is all important-on earth.

Should these same conditions of the space environment prove more rewarding at a later date, in particular for the production of materials of superlative quality, or not feasil le on earth, this will be all the better. But these are only ambitions for the future and as sucb we are wary of placing them too high and of trying to attain them too soon. 